\title{
Intraspecific morphometric variation in myxosporeans
}

\author{
Yanhua Zhai ${ }^{1,2,3}$, Christopher M. Whipps ${ }^{4}$, Zemao Gu ${ }^{1,2,3}$, Qingxiang Guo ${ }^{1}$, Zizhen Wu ${ }^{1}$, Hongmei Wang ${ }^{1}$ and \\ Yang Liu ${ }^{1,2,3}$
}

\author{
${ }^{1}$ Department of Aquatic Animal Medicine, College of Fisheries, Huazhong Agricultural University, Wuhan, People's Republic of \\ China; \\ ${ }^{2}$ Key Lab of Freshwater Animal Breeding, Ministry of Agriculture, Wuhan, People's Republic of China; \\ ${ }^{3}$ Freshwater Aquaculture Collaborative Innovation Center of Hubei Province, Wuhan, People's Republic of China; \\ ${ }^{4}$ State University of New York, College of Environmental Science and Forestry, Environmental and Forest Biology, Syracuse, NY, \\ USA
}

\begin{abstract}
Morphometric data from spores of ten myxosporean species were statistically analysed to explore myxosporean intraspecific variation in measurements when obtained from a sample from: (1) the same plasmodium, (2) different plasmodia from the same host and (3) different host individuals and localities. In some cases, significant differences in spore dimensions were found between samples from the same plasmodium, highlighting the difficulty of obtaining representative measurements of myxosporean spore. In addition, significant differences in spore dimensions were found when plasmodia from the same site of infection were compared, suggesting that measurements of spores should come from several different plasmodia of the sampling to increase the reliability of the morphology data. Moreover, significant differences in spore dimensions were observed for most spore dimensions when data were compared between localities. In all cases, there was clear overlap in ranges of dimensions even when means differed significantly. The present statistical analysis shows that intraspecific morphometric variation of myxosporean species commonly occurs, highlighting the importance of reporting ranges of measurements for a species, not just the mean dimensions, and taking into account all evidence when assigning or describing myxosporean species.
\end{abstract}

Keywords: intraspecific variation, Myxozoa, morphology, species differentiation, Myxobolidae

Traditionally, myxosporean species have been identified principally on the basis of spore morphology, but DNA sequencing data have become important for resolving relationships and determining taxonomic affinities (Atkinson et al. 2015). Given that molecular data on most species are absent, comparison of spore morphology, especially detecting differences in morphometry, is crucial and necessary. However, criteria for what might represent intraspecific and interspecific morphometrics is undefined, making the species identification based on morphometric comparison somewhat subjective.

Often, when a species is collected, the range and mean spore dimensions (usually including spore length, spore width and polar capsule dimensions) can be compared to known species and where overlap occurs there is a candidate species match. Host, geography and tissue infected are other characters that must be considered. When spore dimensions overlap, but other information suggesting distinct species is present, more sophisticated statistical analyses can be performed to determine if statistical differences occur between spore dimensions. For example, Easy et al. (2005) found evidence of two species of Myxobolus Bütschli, 1882 species in the musculature of Percopsis omiscomaycus (Walbaum) based on histology and molecular data. These species had overlapping but significantly different spore lengths, with the intermuscular Myxobolus procerus Kudo, 1934 having longer spores than the intramuscular Myxobolus intramusculi Easy, Johnson et Cone, 2005.

A similar approach was taken by Ferguson et al. (2008) for species of Myxobolus infecting salmon, one found in the peripheral nerves of the musculature and the other intramuscular. The spores of Myxobolus fryeri Ferguson, Atkinson, Whipps et Kent, 2008 from the nerves were significantly shorter than those of the intramuscular Myxobolus insidiosus Wyatt et Pratt, 1963. Sometimes, the evidence is suggestive of distinct species, but not as definitive as the above cases. For example, in a study of global samples of Kudoa thrysites Gilchrist, 1924 and Kudoa histolytica Pérard, 1928, Whipps and Kent (2006) found geographically distinct genetic lineages of the parasite and some statistical differences in spore dimensions, but these were not consistent by site, and a single species was maintained. 
Table 1. List of myxosporeans analysed in the present study.

\begin{tabular}{|c|c|c|c|c|}
\hline Species name & Host & Site of infection & Location/ Accession number* & Date \\
\hline Henneguya doneci Schulman, 1962 & $\begin{array}{l}\text { Carassius auratus gibelio } \\
\text { Bloch }\end{array}$ & gills & Baishazhou - fish market & $2011 / 11$ \\
\hline $\begin{array}{l}\text { Myxobolus ampullicapsulatus Zhao, } \\
\text { Sun, Kent, Deng et Whipps, } 2008\end{array}$ & Carassius auratus gibelio & gills & Honghu City - fish farm & $2012 / 06$ \\
\hline \multirow[t]{2}{*}{ M. koi Kudo, 1919} & Cyprinus carpio Linnaeus & gills & Baishazhou - fish market (location 1)/(KJ725077) & $2011 / 03$ \\
\hline & Cyprinus carpio & gills & Huanong - fish market (location 2)/(KT962988) & $2012 / 03$ \\
\hline \multirow[t]{2}{*}{ M. musseliusae Yakovchuk, 1979} & Cyprinus carpio & gills & Honghu City - fish farm (location 1)/(JQ040301) & $2011 / 03$ \\
\hline & Cyprinus carpio & gills & Baishazhou - fish market (location 2)/(KT962987) & $2012 / 03$ \\
\hline M. nielii $\mathrm{Nie}$ et Li, 1973 & Carassius auratus gibelio & gills & Honghu City - fish farm & $2012 / 06$ \\
\hline M. pseudoacinosus Huang, 2014 & Cyprinus carpio & gills & Baishazhou - fish market & $2014 / 04$ \\
\hline \multirow[t]{2}{*}{ M. tsangwuensis Chen, 1954} & Cyprinus carpio & gills & Baishazhou - fish market (location 1)/(KJ725076) & $2012 / 12$ \\
\hline & Cyprinus carpio & gills & Honghu City - fish farm (location 2)/(KJ561441) & 2013/01 \\
\hline M. turpisrotundus & Carassius auratus gibelio & skin, jaws, gills, fins & Yezhi Lake (location 1)/(GU570996) & $2008 / 12$ \\
\hline Zhang, Wang, Li et Gong, 2010 & Carassius auratus gibelio & skin, jaws, gills, fins & Baoan Lake (location 2)/(KJ725073) & $2011 / 01$ \\
\hline $\begin{array}{l}\text { Thelohanellus kitauei } \\
\text { Egusa et Nakajima, } 1981\end{array}$ & Cyprinus carpio & intestine & Baishazhou - fish market & $2010 / 02$ \\
\hline T. toyamai Kudo, 1915 & Cyprinus carpio & gills & Baishazhou - fish market & $2012 / 03$ \\
\hline
\end{tabular}

* molecular sequence in Genbank of the sample from different host individual and localities.

Heiniger et al. (2013) found genetic and morphological variation in Kudoa whippsi Burger et Adlard, 2010 collected from different hosts and localities, and the authors concluded this likely represents a species complex but maintained the single species until further evidence can clearly distinguish isolates. It is worthwhile noting that the method of fixation or lack of fixation is important to report when documenting species. Fixatives are known to change spore dimensions (Parker and Warner 1970) relative to fresh samples. Optimally fresh material would always be used, but this is not always possible and the way the spores were examined should be clearly reported.

The examples above demonstrate the challenges and innovative approaches for delineating myxosporean species with overlapping spore dimensions. The examples also illustrate that there is no perfect solution when overlap is encountered. Sometimes two or more species are identified, sometimes a single species is maintained. Total evidence must be considered, but we set out to evaluate the relative importance of intraspecific morphological differences between samples of spores for myxobolid species. Here, we examined statistical differences between spore samples of ten species testing for differences between samples from the same plasmodium, different plasmodia, and different localities.

\section{MATERIALS AND METHODS}

Ten myxosporean species (Table 1) were collected from freshwater fish in China from December 2008 to April 2014. All these species have been identified in our former researches (Liu et al. 2010, 2011, 2013, Huang 2014, Huang et al. 2014, Liu 2014) or other ongoing studies (Y.L. - unpublished data). For samples from different fish hosts and localities, we confirmed conspecificity by DNA sequence analysis of small subunit ribosomal DNA sequences obtained in the aforementioned studies. We categorised our data into groups A, B, C and D as follows: (A) spore data from plasmodium 1, fish 1; (B) a second sample from the same plasmodium in A; (C) a sample from a second plasmodium, but the same fish as in A and B; and (D) a sample from a plasmodium from a second fish (different locality). Measurements of spores including spore length (SL), spore width (SW), polar capsule length (PCL) or large polar capsule length (LPCL) and small polar capsule length (SPCL), polar capsule width (PCW) or large polar capsule width (LPCW) and small polar capsule width (SPCW) were performed using an Olympus $\mathrm{BH} 2$ microscope equipped with an ocular micrometre. Mean and standard deviations of each spore dimension were obtained from fresh spores $(n=30)$. All morphology data were statistically analysed for difference using t-tests in software of Statistical Product and Service Solutions (SPASS) 19.0 with $\alpha=0.05$.

\section{RESULTS}

For species where only samples from the same plasmodium were compared (Fig. 1), at least one significant difference was found for all species (Table 2). When the same and different plasmodia were compared (Fig. 2), there were differences in the spore length and width for Thelohanellus toyamai Kudo, 1915 for samples from the same plasmodium (A vs B), for T. toyamai from different plasmodia (A vs C), and a difference in polar capsule width of Henneguya doneci Shulman, 1962 from different plasmodia (B vs C) (Table 2), but no other statistical difference were found. For four species, all comparisons were made (Fig. 3) and several differences were found, especially when dimensions were compared between localities (A vs D, B vs D, C vs D) (Table 2).

\section{DISCUSSION}

The statistical comparisons in the present study indicated that a sample from the same plasmodium might be statistically different from a sample from that same plasmodium as observed in Myxobolus ampullicapsulatus Zhao, Sun, Kent, Deng et Whipps, 2008, Myxobolus musseliusae Yakovchuk, 1979, Myxobolus nielii Nie et Li, 1973, Myxobolus pseudoacinosus Huang, 2014, Myxobolus tsangwuensis Chen, 1954, Thelohanellus kitauei Egusa et Nakajima, 1981 and T. toyamai. Only Myxobolus koi Kudo, 1919, H. doneci and Myxobolus turpisrotundus Zhang, Wang, Li et Gong, 2010 did not show any significant differences between these samples (Table 2). For the intraspecific morphometric variation of myxosporeans from 

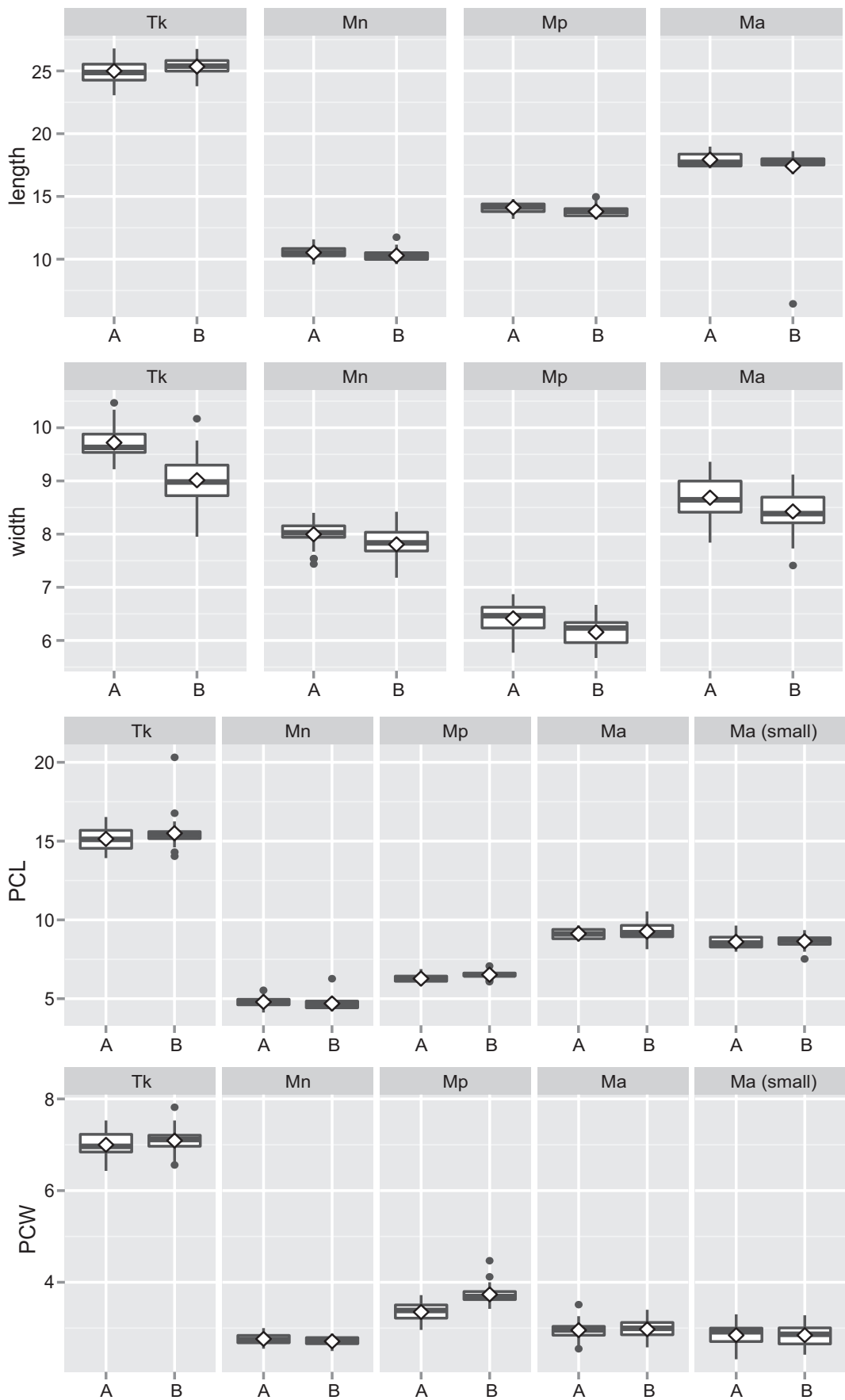

Fig. 1. Boxplot showing comparisons of spore dimensions (in micrometres) for species where two samples from a single plasmodium were made (A $v s \mathrm{~B})$. Boxes show interquartile range, median as a line across the box, mean is indicated with a white diamond, and outliers are black dots. Abbreviations: Tk - Thelohanellus kitauei; Mn - Myxobolus nielii; Mp - Myxobolus pseudoacinosus; Ma - Myxobolus ampullicapsulatus.

different plasmodia (A vs B vs C), significant differences in spore dimension of plasmodium 1 and plasmodium 2 were found for H. doneci (only PCW), M. koi (only PCL), T. toyamai (only SL), M. musseliusae, M. turpisrotundus, and $M$. tsangwuensis (Table 2). Interestingly, we found differences in spore dimensions between different plasmodia for $M$. turpisrotundus (B vs $\mathrm{C}$ ), but not between spore dimensions from samples of the same plasmodium (A vs B). This sort of stochasticity suggests that when measurements are made, they should come from several different plasmo- dia and possibly with a greater sample size to reduce the variation of the morphology data.

For comparisons between localities, there were differences in dimensions for all four species (Table 2). The DNA sequences from different locations were identical within any given species (accession numbers in Table 1), supporting our assumption of conspecificity. The morphological differences are likely representative of the true variation between isolates of the same species, similar to studies on species of Kudoa Meglitsch, 1947 discussed 
Table 2. Spore measurements for length (SL), width (SW), polar capsule length (PCL) and polar capsule width (PCW) for samples from different sources $(\mathrm{n}=30)$. Where two measurements are provided for PCL and PCW, the species had distinctly different size of polar capsules, and both were considered here. Sources are categorised as A - fish 1, plasmodium 1, sample 1; B - same fish and plasmodium as A, sample 2; C - same fish as A and B, different plasmodium; D - fish 2 (different locality), single plasmodium. Pairwise statistical comparisons are shown with statistically significant $\mathrm{p}$ values in bold.

\begin{tabular}{|c|c|c|c|c|c|c|c|c|c|c|c|}
\hline \multicolumn{12}{|c|}{ Sample category } \\
\hline & & $\mathrm{A}$ & $\mathrm{B}$ & $\mathrm{C}$ & $\mathrm{D}$ & $\mathrm{A} v s \mathrm{~B}$ & $\mathrm{~A} v s \mathrm{C}$ & $\mathrm{A} v s \mathrm{D}$ & $\mathrm{B} v s \mathrm{C}$ & $\mathrm{B} v s \mathrm{D}$ & $\mathrm{C} v s \mathrm{D}$ \\
\hline $\begin{array}{l}\text { Myxobolus turpisrotun- } \\
\text { dus }\end{array}$ & SL & $\begin{array}{l}8.60 \pm 0.23 \\
(8.20-9.31)\end{array}$ & $\begin{array}{l}8.69 \pm 0.31 \\
(8.27-9.75)\end{array}$ & $\begin{array}{l}8.46 \pm 0.35 \\
(7.92-9.17)\end{array}$ & $\begin{array}{l}8.47 \pm 0.29 \\
(7.97-9.14)\end{array}$ & 0.216 & 0.085 & 0.065 & 0.010 & 0.006 & 0.939 \\
\hline \multirow[t]{3}{*}{$\begin{array}{l}\text { Zhang, Wang, Li et } \\
\text { Gong, } 2010\end{array}$} & SW & $\begin{array}{l}8.21 \pm 0.23 \\
(7.75-8.66)\end{array}$ & $\begin{array}{l}8.36 \pm 0.34 \\
(7.92-9.25)\end{array}$ & $\begin{array}{l}8.11 \pm 0.26 \\
(7.65-8.57)\end{array}$ & $\begin{array}{l}7.88 \pm 0.34 \\
(7.31-8.47)\end{array}$ & 0.061 & 0.180 & 0.000 & 0.003 & 0.000 & 0.004 \\
\hline & PCL & $\begin{array}{l}4.16 \pm 0.19 \\
(3.68-4.49)\end{array}$ & $\begin{array}{l}4.11 \pm 0.22 \\
(3.74-4.49)\end{array}$ & $\begin{array}{l}4.15 \pm 0.17 \\
(3.90-4.48)\end{array}$ & $\begin{array}{l}4.32 \pm 0.15 \\
(4.03-4.62)\end{array}$ & 0.340 & 0.768 & 0.001 & 0.462 & 0.000 & 0.000 \\
\hline & PCW & $\begin{array}{l}3.17 \pm 0.15 \\
(2.78-3.37)\end{array}$ & $\begin{array}{l}3.19 \pm 0.11 \\
(2.94-3.42)\end{array}$ & $\begin{array}{l}3.19 \pm 0.13 \\
(2.93-3.43)\end{array}$ & $\begin{array}{l}3.06 \pm 0.13 \\
(2.75-3.31)\end{array}$ & 0.522 & 0.465 & 0.007 & 0.876 & 0.000 & 0.000 \\
\hline \multirow[t]{4}{*}{$\begin{array}{l}\text { Myxobolus tsangwuensis } \\
\text { Chen, } 1954\end{array}$} & SL & $\begin{array}{l}10.36 \pm 0.40 \\
(9.77-11.25)\end{array}$ & $\begin{array}{l}10.39 \pm 0.32 \\
(9.74-11.02)\end{array}$ & $\begin{array}{l}10.90 \pm 0.51 \\
(9.72-11.92)\end{array}$ & $\begin{array}{c}9.92 \pm 0.51 \\
(8.87-11.13)\end{array}$ & 0.790 & 0.000 & 0.000 & 0.000 & 0.000 & 0.000 \\
\hline & SW & $\begin{array}{l}8.50 \pm 0.30 \\
(7.80-8.88)\end{array}$ & $\begin{array}{l}8.42 \pm 0.22 \\
(7.89-8.88)\end{array}$ & $\begin{array}{l}9.28 \pm 0.44 \\
(8.34-9.97)\end{array}$ & $\begin{array}{l}8.32 \pm 0.35 \\
(7.62-9.03)\end{array}$ & 0.279 & 0.000 & 0.042 & 0.000 & 0.185 & 0.000 \\
\hline & PCL & $\begin{array}{l}4.57 \pm 0.20 \\
(4.10-4.88) \\
3.25 \pm 0.20 \\
(2.94-3.92)\end{array}$ & $\begin{array}{l}4.94 \pm 0.22 \\
(4.28-5.51) \\
3.64 \pm 0.23 \\
(3.22-4.25)\end{array}$ & $\begin{array}{l}4.39 \pm 0.25 \\
(4.40-5.31) \\
3.93 \pm 0.26 \\
(3.43-4.48)\end{array}$ & $\begin{array}{l}4.50 \pm 0.26 \\
(4.07-5.09) \\
3.49 \pm 0.29 \\
(3.30-4.05)\end{array}$ & $\begin{array}{l}0.000 \\
0.000\end{array}$ & $\begin{array}{l}\mathbf{0 . 0 0 0} \\
\mathbf{0 . 0 0 0}\end{array}$ & $\begin{array}{l}0.287 \\
\mathbf{0 . 0 0 1}\end{array}$ & $\begin{array}{l}0.859 \\
\mathbf{0 . 0 0 0}\end{array}$ & $\begin{array}{c}0.000 \\
0.028\end{array}$ & $\begin{array}{l}\mathbf{0 . 0 0 0} \\
\mathbf{0 . 0 0 0}\end{array}$ \\
\hline & PCW & $\begin{array}{l}2.90 \pm 0.20 \\
(2.49-3.22) \\
2.18 \pm 0.23 \\
(1.35-2.52)\end{array}$ & $\begin{array}{l}3.28 \pm 0.19 \\
(2.77-3.62) \\
2.43 \pm 0.14 \\
(2.17-2.75)\end{array}$ & $\begin{array}{l}3.31 \pm 0.19 \\
(2.89-3.63) \\
2.59 \pm 0.17 \\
(2.30-2.97)\end{array}$ & $\begin{array}{l}3.07 \pm 0.20 \\
(2.69-3.60) \\
2.42 \pm 0.16 \\
(2.11-2.76)\end{array}$ & $\begin{array}{l}0.000 \\
0.000\end{array}$ & $\begin{array}{l}\mathbf{0 . 0 0 0} \\
\mathbf{0 . 0 0 0}\end{array}$ & $\begin{array}{l}\mathbf{0 . 0 0 2} \\
\mathbf{0 . 0 0 0}\end{array}$ & $\begin{array}{l}0.540 \\
\mathbf{0 . 0 0 0}\end{array}$ & $\begin{array}{l}\mathbf{0 . 0 0 0} \\
0.735\end{array}$ & $\begin{array}{l}\mathbf{0 . 0 0 0} \\
\mathbf{0 . 0 0 0}\end{array}$ \\
\hline \multirow[t]{4}{*}{$\begin{array}{l}\text { Myxobolus koi Kudo, } \\
1919\end{array}$} & $\mathrm{SL}$ & $\begin{array}{l}12.93 \pm 0.54 \\
(11.58-13.74)\end{array}$ & $\begin{array}{c}13.12 \pm 0.54 \\
(11.97-14.01)\end{array}$ & $\begin{array}{l}13.11 \pm 0.50 \\
(12.01-14.05)\end{array}$ & $\begin{array}{c}12.77 \pm 0.79 \\
(10.61-13.80)\end{array}$ & 0.176 & 0.178 & 0.368 & 0.951 & 0.049 & 0.049 \\
\hline & SW & $\begin{array}{l}7.35 \pm 0.29 \\
(6.90-8.01)\end{array}$ & $\begin{array}{l}7.48 \pm 0.35 \\
(6.46-8.09)\end{array}$ & $\begin{array}{l}7.42 \pm 0.24 \\
(6.74-7.84)\end{array}$ & $\begin{array}{l}6.74 \pm 0.32 \\
(5.94-7.39)\end{array}$ & 0.123 & 0.277 & 0.000 & 0.483 & 0.000 & 0.000 \\
\hline & PCL & $\begin{array}{l}7.02 \pm 0.30 \\
(6.46-7.64)\end{array}$ & $\begin{array}{l}7.12 \pm 0.38 \\
(6.15-7.81)\end{array}$ & $\begin{array}{l}7.21 \pm 0.31 \\
(6.46-7.79)\end{array}$ & $\begin{array}{l}6.70 \pm 0.38 \\
(6.06-7.65)\end{array}$ & 0.295 & 0.022 & 0.001 & 0.303 & 0.000 & 0.000 \\
\hline & PCW & $\begin{array}{l}2.59 \pm 0.24 \\
(1.98-2.93)\end{array}$ & $\begin{array}{l}2.60 \pm 0.16 \\
(2.29-2.90)\end{array}$ & $\begin{array}{l}2.63 \pm 0.14 \\
(2.38-2.94)\end{array}$ & $\begin{array}{l}2.48 \pm 0.14 \\
(2.23-2.79)\end{array}$ & 0.782 & 0.402 & 0.035 & 0.465 & 0.002 & 0.000 \\
\hline \multirow[t]{4}{*}{$\begin{array}{l}\text { Myxobolus musseliusae } \\
\text { Yakovchuk, } 1979\end{array}$} & $\mathrm{SL}$ & $\begin{array}{c}11.75 \pm 0.51 \\
(10.76-12.98)\end{array}$ & $\begin{array}{c}12.96 \pm 0.58 \\
(11.53-13.71)\end{array}$ & $\begin{array}{c}11.69 \pm 0.56 \\
(10.76-13.10)\end{array}$ & $\begin{array}{l}10.75 \pm 0.40 \\
(9.65-11.56)\end{array}$ & 0.000 & 0.660 & 0.000 & 0.000 & 0.000 & 0.000 \\
\hline & SW & $\begin{array}{c}9.52 \pm 0.75 \\
(8.71-11.78)\end{array}$ & $\begin{array}{l}10.80 \pm 0.41 \\
(9.96-11.65)\end{array}$ & $\begin{array}{c}9.80 \pm 0.48 \\
(8.89-10.59)\end{array}$ & $\begin{array}{l}9.25 \pm 0.35 \\
(8.25-9.73)\end{array}$ & 0.000 & 0.096 & 0.079 & 0.000 & 0.000 & 0.000 \\
\hline & PCL & $\begin{array}{l}5.07 \pm 0.34 \\
(4.45-5.67) \\
3.95 \pm 0.29 \\
(3.16-4.59)\end{array}$ & $\begin{array}{l}5.37 \pm 0.47 \\
(4.49-6.16) \\
4.03 \pm 0.34 \\
(3.40-4.62)\end{array}$ & $\begin{array}{l}4.98 \pm 0.23 \\
(4.51-5.44) \\
3.91 \pm 0.26 \\
(3.12-4.22)\end{array}$ & $\begin{array}{l}4.75 \pm 0.22 \\
(4.35-5.15) \\
3.38 \pm 0.26 \\
(2.94-3.88)\end{array}$ & $\begin{array}{l}\mathbf{0 . 0 0 6} \\
0.276\end{array}$ & $\begin{array}{l}0.276 \\
0.655\end{array}$ & $\begin{array}{l}0.000 \\
0.000\end{array}$ & $\begin{array}{l}\mathbf{0 . 0 0 0} \\
0.127\end{array}$ & $\begin{array}{l}0.000 \\
0.000\end{array}$ & $\begin{array}{l}0.000 \\
0.000\end{array}$ \\
\hline & PCW & $\begin{array}{l}3.27 \pm 0.15 \\
(2.96-3.56) \\
2.76 \pm 0.15 \\
(2.53-3.13)\end{array}$ & $\begin{array}{l}3.51 \pm 0.36 \\
(2.85-4.05) \\
2.67 \pm 0.24 \\
(2.39-3.53)\end{array}$ & $\begin{array}{l}3.45 \pm 0.38 \\
(2.98-4.95) \\
2.69 \pm 0.18 \\
(2.22-2.95)\end{array}$ & $\begin{array}{l}3.19 \pm 0.17 \\
(2.82-3.47) \\
2.34 \pm 0.14 \\
(2.08-2.67)\end{array}$ & $\begin{array}{l}0.001 \\
0.096\end{array}$ & $\begin{array}{l}0.019 \\
0.107\end{array}$ & $\begin{array}{l}0.073 \\
\mathbf{0 . 0 0 0}\end{array}$ & $\begin{array}{l}0.557 \\
0.737\end{array}$ & $\begin{array}{l}0.000 \\
0.000\end{array}$ & $\begin{array}{l}0.001 \\
0.000\end{array}$ \\
\hline \multirow[t]{4}{*}{$\begin{array}{l}\text { Henneguya doneci } \\
\text { Shulman, } 1962\end{array}$} & $\mathrm{SL}$ & $\begin{array}{l}8.81 \pm 0.48 \\
(7.63-9.65)\end{array}$ & $\begin{array}{l}8.63 \pm 0.51 \\
(7.54-9.58)\end{array}$ & $\begin{array}{l}8.81 \pm 0.51 \\
(7.81-9.73)\end{array}$ & - & 0.162 & 0.992 & - & 0.177 & - & - \\
\hline & SW & $\begin{array}{l}8.33 \pm 0.38 \\
(7.69-9.07)\end{array}$ & $\begin{array}{l}8.24 \pm 0.39 \\
(7.32-8.90)\end{array}$ & $\begin{array}{l}8.39 \pm 0.27 \\
(7.94-9.02)\end{array}$ & - & 0.361 & 0.500 & - & 0.090 & - & - \\
\hline & PCL & $\begin{array}{l}5.03 \pm 0.30 \\
(4.40-5.60)\end{array}$ & $\begin{array}{l}4.99 \pm 0.28 \\
(4.41-5.50)\end{array}$ & $\begin{array}{l}4.89 \pm 0.29 \\
(4.32-5.40)\end{array}$ & - & 0.636 & 0.084 & - & 0.184 & - & - \\
\hline & PCW & $\begin{array}{l}3.21 \pm 0.20 \\
(2.76-3.63)\end{array}$ & $\begin{array}{l}3.27 \pm 0.14 \\
(2.90-3.59)\end{array}$ & $\begin{array}{l}3.12 \pm 0.19 \\
(2.79-3.46)\end{array}$ & - & 0.179 & 0.087 & - & 0.001 & - & - \\
\hline \multirow[t]{4}{*}{$\begin{array}{l}\text { Thelohanellus toyamai } \\
\text { Kudo, } 1915\end{array}$} & SL & $\begin{array}{c}15.07 \pm 0.56 \\
(14.07-16.45)\end{array}$ & $\begin{array}{c}15.52 \pm 0.52 \\
(14.65-16.76)\end{array}$ & $\begin{array}{c}15.36 \pm 0.44 \\
(14.40-16.20)\end{array}$ & - & 0.002 & 0.034 & - & 0.202 & - & - \\
\hline & SW & $\begin{array}{l}5.39 \pm 0.22 \\
(4.97-5.89)\end{array}$ & $\begin{array}{l}5.20 \pm 0.37 \\
(3.80-5.72)\end{array}$ & $\begin{array}{l}5.35 \pm 0.26 \\
(4.79-5.85)\end{array}$ & - & 0.020 & 0.582 & - & 0.069 & - & - \\
\hline & PCL & $\begin{array}{l}7.18 \pm 0.34 \\
(6.40-7.81)\end{array}$ & $\begin{array}{l}7.12 \pm 0.30 \\
(6.30-7.75)\end{array}$ & $\begin{array}{l}7.21 \pm 0.34 \\
(6.34-7.89)\end{array}$ & - & 0.453 & 0.742 & - & 0.274 & - & - \\
\hline & PCW & $\begin{array}{l}3.35 \pm 0.20 \\
(2.83-3.71) \\
\end{array}$ & $\begin{array}{l}3.38 \pm 0.15 \\
(3.17-3.67) \\
\end{array}$ & $\begin{array}{l}3.29 \pm 0.24 \\
(2.80-3.81)\end{array}$ & - & 0.552 & 0.295 & - & 0.096 & - & - \\
\hline \multirow[t]{4}{*}{$\begin{array}{l}\text { Myxobolus nielii } \\
\text { Nie et Li, } 1973\end{array}$} & $\mathrm{SL}$ & $\begin{array}{l}10.52 \pm 0.43 \\
(9.58-11.58)\end{array}$ & $\begin{array}{l}10.29 \pm 0.45 \\
(9.60-11.76)\end{array}$ & - & - & 0.045 & - & - & - & - & - \\
\hline & SW & $\begin{array}{l}8.00 \pm 0.23 \\
(7.44-8.40)\end{array}$ & $\begin{array}{l}7.81 \pm 0.31 \\
(7.18-8.42)\end{array}$ & - & - & 0.009 & - & - & - & - & - \\
\hline & PCL & $\begin{array}{l}4.79 \pm 0.33 \\
(4.12-5.53)\end{array}$ & $\begin{array}{l}4.69 \pm 0.39 \\
(4.20-6.27)\end{array}$ & - & - & 0.287 & - & - & - & - & - \\
\hline & PCW & $\begin{array}{l}2.76 \pm 0.10 \\
(2.55-3.00)\end{array}$ & $\begin{array}{l}2.71 \pm 0.10 \\
(2.50-2.88)\end{array}$ & - & - & 0.053 & - & - & - & - & - \\
\hline
\end{tabular}


Table 2. Continued.

\begin{tabular}{|c|c|c|c|c|c|c|c|c|c|c|c|}
\hline \multicolumn{12}{|c|}{ Sample category } \\
\hline & & A & B & $\mathrm{C}$ & $\mathrm{D}$ & $\mathrm{A} v s \mathrm{~B}$ & $\mathrm{~A} v s \mathrm{C}$ & $\mathrm{A} v s \mathrm{D}$ & $\mathrm{B} v s \mathrm{C}$ & $\mathrm{B} v s \mathrm{D}$ & $\mathrm{C} v s \mathrm{D}$ \\
\hline \multirow[t]{4}{*}{$\begin{array}{l}\text { Thelohanellus kitauei } \\
\text { Egusa et Nakajima, } 1981\end{array}$} & SL & $\begin{array}{c}24.99 \pm 1.09 \\
(23.06-26.79)\end{array}$ & $\begin{array}{c}25.35 \pm 0.82 \\
(23.78-26.75)\end{array}$ & - & - & 0.158 & - & - & - & - & - \\
\hline & SW & $\begin{array}{c}9.72 \pm 0.31 \\
(9.22-10.47)\end{array}$ & $\begin{array}{c}9.01 \pm 0.43 \\
(7.95-10.17)\end{array}$ & - & - & 0.000 & - & - & - & - & - \\
\hline & PCL & $\begin{array}{c}15.15 \pm 0.68 \\
(13.92-16.53)\end{array}$ & $\begin{array}{c}15.50 \pm 1.06 \\
(14.05-20.32)\end{array}$ & - & - & 0.122 & - & - & - & - & - \\
\hline & PCW & $\begin{array}{l}7.00 \pm 0.26 \\
(6.43-7.53)\end{array}$ & $\begin{array}{l}7.09 \pm 0.26 \\
(6.56-7.82)\end{array}$ & - & - & 0.169 & - & - & - & - & - \\
\hline \multirow[t]{4}{*}{$\begin{array}{l}\text { Myxobolus pseudoacino- } \\
\text { sus Huang, } 2014\end{array}$} & SL & $\begin{array}{c}14.11 \pm 0.38 \\
(13.21-14.79)\end{array}$ & $\begin{array}{c}13.80 \pm 0.44 \\
(13.15-14.98)\end{array}$ & - & - & 0.005 & - & - & - & - & - \\
\hline & SW & $\begin{array}{l}6.42 \pm 0.29 \\
(5.77-6.87)\end{array}$ & $\begin{array}{l}6.16 \pm 0.27 \\
(5.67-6.67)\end{array}$ & - & - & 0.001 & - & - & - & - & - \\
\hline & PCL & $\begin{array}{l}6.28 \pm 0.27 \\
(5.80-6.88)\end{array}$ & $\begin{array}{l}6.52 \pm 0.20 \\
(6.07-7.07)\end{array}$ & - & - & 0.000 & - & - & - & - & - \\
\hline & PCW & $\begin{array}{l}3.35 \pm 0.20 \\
(2.96-3.72)\end{array}$ & $\begin{array}{l}3.74 \pm 0.21 \\
(3.42-4.47)\end{array}$ & - & - & 0.000 & - & - & - & - & - \\
\hline $\begin{array}{l}\text { Myxobolus ampullicap- } \\
\text { sulatus }\end{array}$ & SL & $\begin{array}{c}17.93 \pm 0.56 \\
(17.24-18.97)\end{array}$ & $\begin{array}{c}17.82 \pm 0.49 \\
(16.77-18.61)\end{array}$ & - & - & 0.203 & - & - & - & - & - \\
\hline \multirow[t]{5}{*}{$\begin{array}{l}\text { Zhao, Sun, Kent, Deng et } \\
\text { Whipps, } 2008\end{array}$} & SW & $\begin{array}{l}8.69 \pm 0.38 \\
(7.84-9.36)\end{array}$ & $\begin{array}{l}8.43 \pm 0.41 \\
(7.41-9.12)\end{array}$ & - & - & 0.013 & - & - & - & - & - \\
\hline & LPCL & $\begin{array}{l}9.13 \pm 0.33 \\
(8.64-9.65)\end{array}$ & $\begin{array}{c}9.26 \pm 0.55 \\
(8.14-10.54)\end{array}$ & - & - & 0.269 & - & - & - & - & - \\
\hline & SPCL & $\begin{array}{l}8.61 \pm 0.41 \\
(7.98-9.64)\end{array}$ & $\begin{array}{l}8.64 \pm 0.42 \\
(7.53-9.35)\end{array}$ & - & - & 0.757 & - & - & - & - & - \\
\hline & LPCW & $\begin{array}{l}2.95 \pm 0.20 \\
(2.55-3.51)\end{array}$ & $\begin{array}{l}2.98 \pm 0.21 \\
(2.58-3.40)\end{array}$ & - & - & 0.708 & - & - & - & - & - \\
\hline & SPCW & $\begin{array}{l}2.85 \pm 0.25 \\
(2.32-3.30)\end{array}$ & $\begin{array}{l}2.85 \pm 0.26 \\
(2.42-3.28)\end{array}$ & - & - & 1.000 & - & - & - & - & - \\
\hline
\end{tabular}
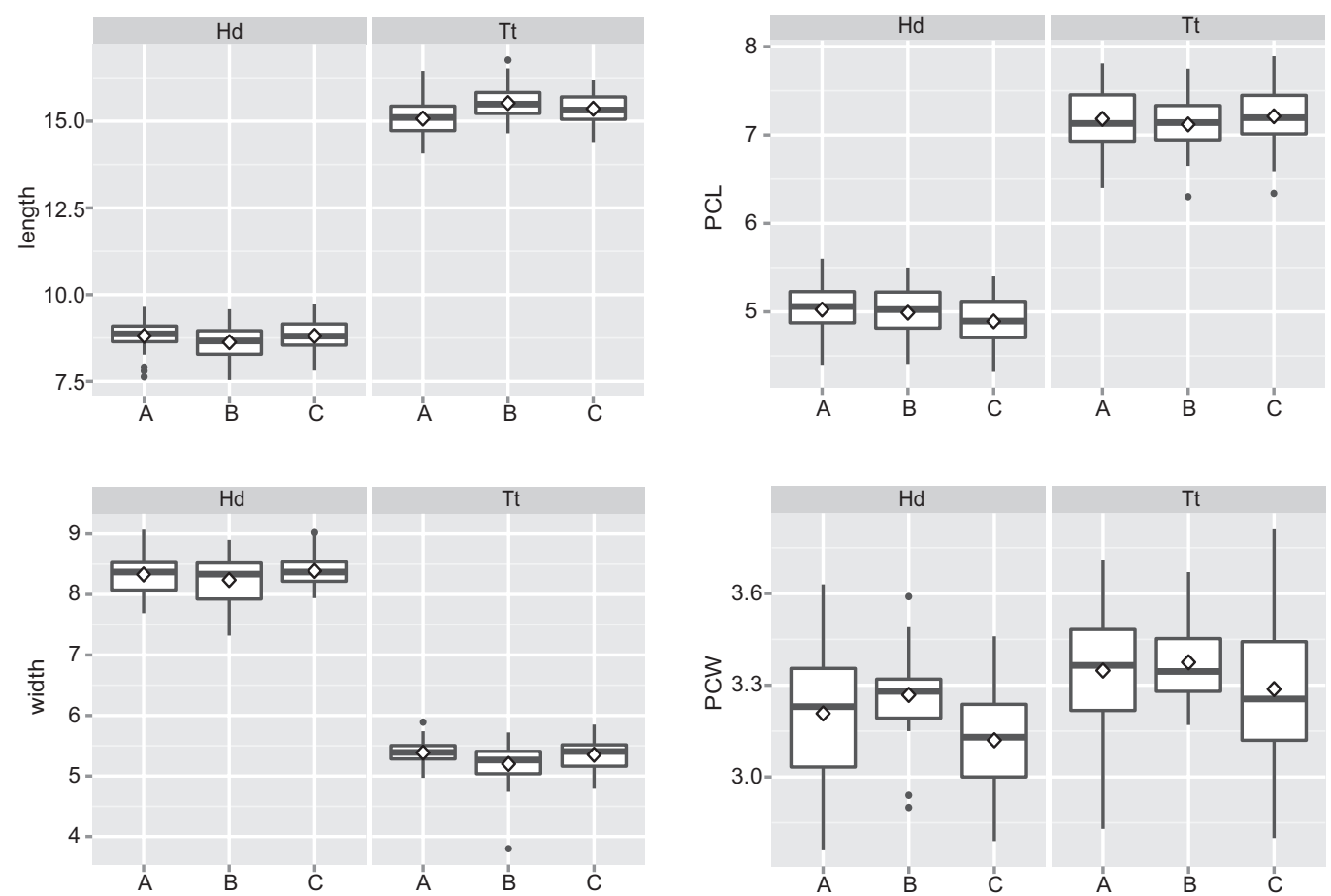

Fig. 2. Boxplot showing comparisons of spore dimensions for species where two samples from a single plasmodium were made and these were also compared to a sample from another plasmodium (A vs B vs C). Boxes show interquartile range, median as a line across the box, mean is indicated with a white diamond, and outliers are black dots. Abbreviations: Hd - Henneguya doneci; $\mathrm{Tt}$ - Thelohanellus toyamai.

above (Whipps and Kent 2006, Heiniger et al. 2013). Intraspecific variation has also been found in actinospores from different localities and hosts (Hallett et al. 2004, Eszterbauer et al. 2006). These differences are potentially the most challenging to deal with because they may not represent statistical anomalies, but actual differences between geographic isolates, and calls to question where and how decisions on intra- and inter-specific difference are made. 

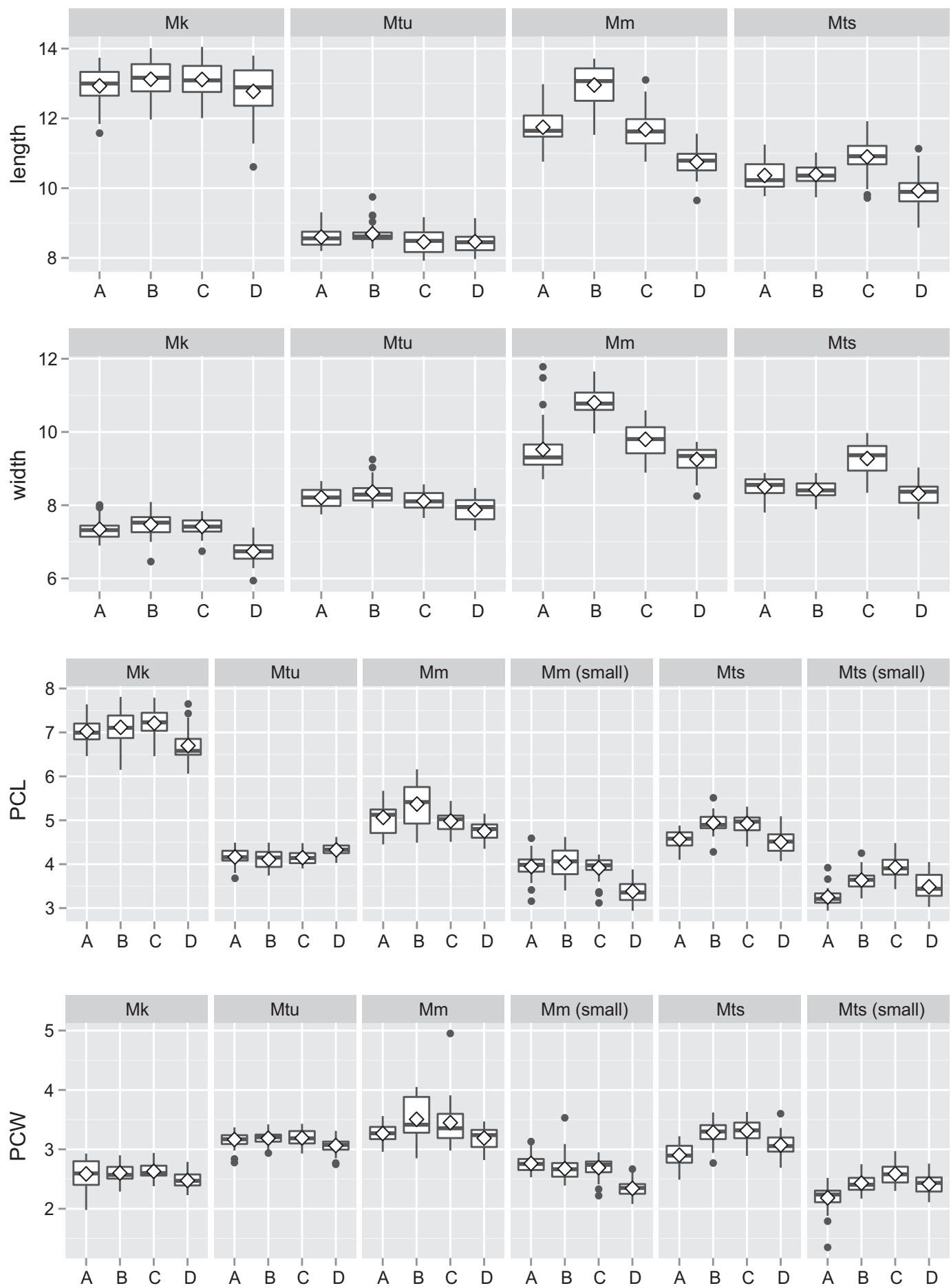

Fig. 3. Boxplot showing comparisons of spore dimensions for species where two samples from a single plasmodium were made, another plasmodium from the same fish was compared to these, and other sample from a fish at a different locality was also compared (A $v s$ B vs $\mathrm{C} v s \mathrm{D}$ ). Boxes show interquartile range, median as a line across the box, mean is indicated with a white diamond, and outliers are black dots. Abbreviations: Mk - Myxobolus koi; Mtu - Myxobolus turpisrotundus; Mm - Myxobolus musseliusae; Mts - Myxobolus tsangwuensis.

Regardless, we have demonstrated that when comparisons are made between samples of spores from the same plasmodium, same fish and distinct host, there are sometimes statistical differences in spore dimensions. This emphasises the importance of considering the range of dimensions for overlap, host species, tissue type and DNA sequence if available, when identifying species or describing new ones.
Acknowledgements. This study was supported by the Nature Science Foundation of China (Projects Nos. 31502209, 31572233, 31501848), New Century Excellent Talents in University (Project No. NCET-12-0866), the Fundamental Research Funds for the Central Universities (Projects Nos. 2662015PY089, 2662014BQ034, 2662015QC027), and Innovation Training Plan of University Student (No. 201510504049). 


\section{REFERENCES}

Atkinson S.D., Bartošová-Sojková P., Whipps C.M. 2015: Approaches for characterizing myxozoan species. In: B. Okamura, A. Gruhl and J.L. Bartholomew (Eds.), Myxozoan Evolution, Ecology and Development. Springer, Berlin, pp. 111-123.

Easy R.H., Johnson S.C., Cone D.K. 2005: Morphological and molecular comparison of Myxobolus procerus (Kudo, 1934) and M. intramusculi n. sp. (Myxozoa) parasitising muscles of the trout-perch Percopsis omiscomaycus. Syst. Parasitol. 61: $115-122$.

Eszterbauer E., Marton S., Rácz O.Z., Letenyei M., MolNÁR K. 2006: Morphological and genetic differences among actinosporean stages of fish-parasitic myxosporeans (Myxozoa) difficulties of species identification. Syst. Parasitol. 65: 97-114.

Ferguson J.A., Atkinson S.D., Whipps C.M., Kent M.L. 2008: Molecular and morphological analysis of Myxobolus spp. of salmonid fishes with the description of Myxobolus fryeri $\mathrm{n}$. $\mathrm{sp}$. J. Parasitol. 94: 1322-1334.

Hallett S.L., Atkinson S.D., Erséus C., El-Matbouli M. 2004: Molecular methods clarify morphometric variation in triactinomyxon spores (Myxozoa) released from different oligochaete hosts. Syst. Parasitol. 57: 1-14.

Heiniger H., Cribb T.H., Adlard R.D. 2013: Intra-specific variation of Kudoa spp. (Myxosporea: Multivalvulida) from apogonid fishes (Perciformes), including the description of two new species, K. cheilodipteri n. sp. and K. cookii n. sp., from Australian waters. Syst. Parasitol. 84: 193-215.

Huang M.J. 2014: Taxonomic study of 4 Myxobolus spp. from the common carp (Cyprinus carpio). PhD thesis, Huazhong Agricultural University, Wuhan, $55 \mathrm{pp}$.

Cite this article as: Zhai Y., Whipps C.M., Gu Z., Guo Q., Wu Z., Wang H., Liu Y. 2016: Intraspecific morphometric variation in myxosporeans. Folia Parasitol. 63: 011. 